\section{MYDENTIST LAUNCHES ITS ATTACK ON PLAQUE WITH APP GAME FOR CHILDREN: PLAQUE ATTACK}

mydentist, the largest provider of dentistry in the UK, has launched a free app-based game as part of its Kid's Club initiative to educate children on the benefits of maintaining good oral health and clarify what foods are good and bad for your teeth.

The mydentist Plaque Attack app-based game, which is available free to download from Apple and Android, challenges users aged five years and upwards to defend their teeth from cavity, decay and plaque causing nasties by identifying food baddies and goodies in a race against the clock - fun for the whole family.

Additional features of the two minute app-based game include:

- Interactive colouring sheets for younger children, reinforced with dental care tips for parents.

- Dental health fact sheets- these include essential information on how to look after your child's smile - from mums-to-be, new-borns and toddlers, right through to bigger boys and girls.

The launch of the educational app follows research findings from 2,000 UK families by mydentist which reveal that when it comes to identifying the good foods from the bad there is a lot of confusion, particularly where sugar content is concerned. Twenty per cent of parents believe fruit smoothies are good for their children's teeth and a further nineteen per cent consider sippy cups to be safe options despite both often containing large amounts of added sugar and additives.

The same research also revealed that $41 \%$ of children don't brush their teeth twice daily and 19\% of parents don't understand the benefits of fluoride.

For more information visit www.mydentist.co.uk/ theplaqueattack.

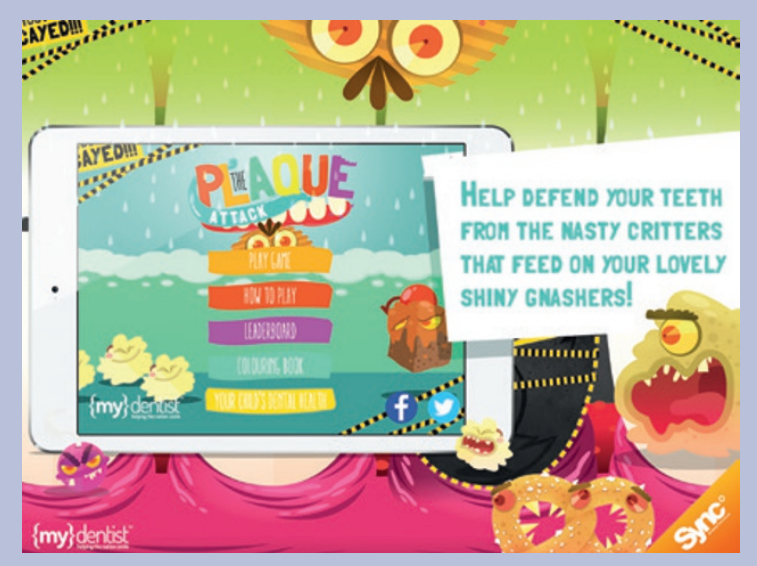

\section{THE NEXT GENERATION IN BULK FILLING REACHES THE UK}

With SonicFill, Kerr provided clinicians with the fast and easy composite filling system for posterior restorations. And now there's SonicFill 2, which enables clinicians to perform posterior restorations with an easy-to-use, one-step procedure that provides everything you need for reliable bulk filling: the adaptation of a flowable composite during placement, a high depth-of-cure, low polymerisation shrinkage stress, and the strength and aesthetics you would expect from a posterior restorative.

SonicFill 2's composite incorporates a highly-filled proprietary resin with special modifiers that react to sonic energy. As sonic energy is applied through the handpiece, the modifier causes the viscosity to drop

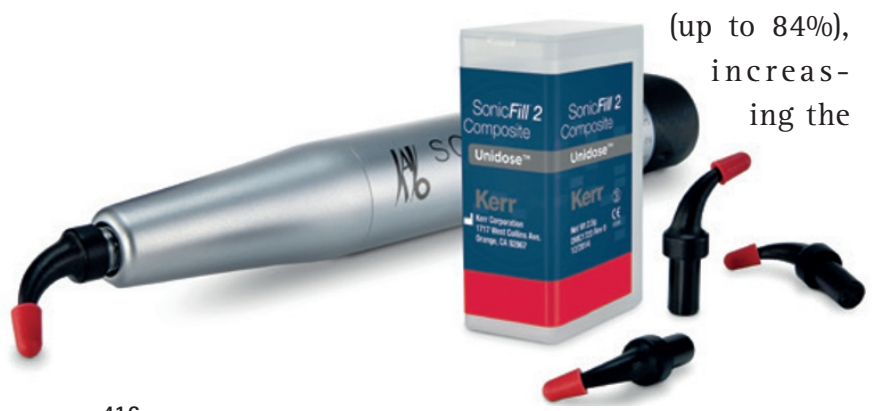

flowability of the composite, enabling quick placement and precise adaptation to the cavity walls. When the sonic energy is stopped, the composite returns to a more viscous, non-slumping state that is perfect for carving and contouring.

The result is:

- No voids, gaps or seams

- Excellent marginal integrity

- Outstanding adaptation to cavity surfaces

- High depth of cure

- Excellent strength, polishability and wear resistance

- Low sensitivity to ambient light.

SonicFill's technology is unlike any other composite material on the market. To experience why for yourself, call 01733 892292, email kerruk@kerrhawe.com or visit www.kerrdental.co.uk.

\section{NO COMPROMISE}

With an in-depth understanding of the daily demands faced by modern clinicians, Carestream Dental offers an array of flexible solutions to suit the way you work.

The CS Adapt module allows you to define and customise images in whatever way you wish.

Up to six pre-set filter options are available for you to choose between, enabling each image to be presented in a format that best suits the individual case.

The intuitive library-style programme facilitates easy browsing, and image selection and the choice of filters ensures faster and more accurate diagnostics every time.

Available to use with various imaging solutions from Carestream Dental, including the CS 8100, CS 8100 3D, CS 9000 and CS 9300 systems, CS Adapt image processing is ideal in a wide variety of situations.

What's more, you can be confident that the customer service delivered by Carestream Dental will be second-to-none, enabling you to make the very most of your innovations.

Don't compromise the way you work when employing new systems - discover CS Adapt.

For more information, contact Carestream Dental on 0800169 9692 or visit www.carestreamdental.co.uk.

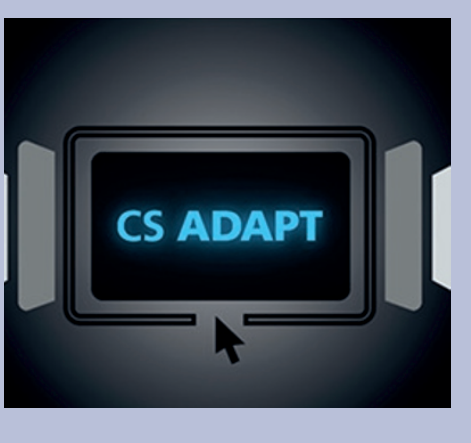

